\title{
One Dimensional Signal Denoising Based on Wavelet Transform and Threshold Theory
}

\author{
Jinming $\mathrm{Fu}^{1}$, Yuanfan $\mathrm{Li}^{2}$, * \\ ${ }^{1}$ College of Science, Huazhong Agricultural University, 430070, Wuhan, China \\ ${ }^{2}$ School of Foreign Languages ,Wuhan Textile University ,430073, Wuhan, China \\ *Corresponding author: mrfu@mail.hzau.edu.cn
}

\begin{abstract}
Wavelet analysis plays an important role in signal processing because of its good time-frequency locality and multi-resolution analysis performance. Based on wavelet analysis in one-dimensional signal denoising are studied, and the principle of threshold and threshold function of wavelet threshold denoising, the selection of wavelet base, decomposition layers and other related issues, and the relevant numerical experiments with Matlab software.
\end{abstract}

Keywords: Wavelet analysis, De-noising, multiresolution analysis, Thresholding Value

\section{Introduction}

Usually the wavelet coefficients after wavelet transform, the wavelet coefficients of signal to noise is greater than the main idea, so wavelet threshold denoising is to find a suitable threshold value as a threshold, when the wavelet coefficient is greater than the threshold, that the coefficient characterizes the signal; when the wavelet coefficient is less than the threshold, it is assumed that the noise coefficient, so it can be separated by the signal and noise threshold. Wavelet threshold denoising is the key to quantify the wavelet coefficients, suitable for white noise removal signal processing, can effectively remove the noise, and retain the signal characteristic spikes.

\section{Wavelet threshold denoising related problems}

\subsection{Wavelet threshold denoising steps}

1) decomposition: $N$ layer decomposition of signal.

2) threshold processing: select a suitable threshold and compare it with the wavelet coefficients after signal transformation. The wavelet coefficients less than the threshold are considered to be noise.

3) reconstruction: the denoised signal can be obtained by reconstructing the processed wavelet coefficients.

\subsection{Selection of wavelet bases}

The wavelet function has orthogonality, symmetry, compact support and other properties, of course, different wavelet functions have different properties, and with different wavelet bases to decompose the signal decomposition results are different, so the denoising effect will vary. So according to the signal characteristics to choose the appropriate wavelet basis function is the primary task of wavelet denoising, in order to reduce the computation decomposition and reconstruction and possible fidelity signal, ideally in signal de-noising in general with orthogonality, symmetry, support small length wavelet.

\subsection{Decomposition layer selection}

The final effect is also related to the selection of decomposition level of wavelet threshold denoising, usually thought to be better denoising effect decomposition more, because the decomposition of more layers, the signal and noise have different features more obvious, which is conducive to the separation of signal and noise. However, the excessive number of decomposition will cause the increase of calculation, and will lead to the error of reconstruction is too large, so that the reconstruction of the signal distortion, so the decomposition of the general layer according to the actual characteristics of the signal to determine. 


\subsection{Threshold selection}

In the process of wavelet threshold denoising, threshold selection is also a direct and important influence factor. Select a different threshold will cause different denoising effect, the threshold of the main general threshold (VisuShrink threshold), rigrsure threshold (SureShrink threshold), maximum threshold (Minimax), BayeShrink threshold, the most commonly used is the universal threshold value, is the threshold by numerical experiments in this paper. In general, the threshold is proposed by Donoho, is generally defined as:

$$
t=\sigma \sqrt{2 \ln N},
$$

Which $\sigma$ is the standard deviation of the noise, $N$ is the signal length.

\subsection{Threshold function selection}

Wavelet transform commonly used threshold function has the following two.

(1)hard threshold function (Hard threshold):

$$
\hat{t}_{j, k}=\left\{\begin{array}{cc}
t_{j, k} & \left|t_{j, k}\right| \geq \lambda \\
0 & \left|t_{j, k}\right|<\lambda
\end{array}\right.
$$

Which $\hat{t}_{j, k}$ represents the processed wavelet coefficients, $t_{j, k}$ represents the wavelet coefficients after wavelet transform, $\lambda$ represents the threshold.

(2)soft threshold function (Soft threshold):

$$
\hat{t}_{j, k}=\left\{\begin{array}{cl}
t_{j, k}-\lambda \operatorname{sgn}\left(t_{j, k}\right) & \left|t_{j, k}\right| \geq \lambda \\
0 & \left|t_{j, k}\right|<\lambda
\end{array}\right.
$$

\section{Numerical experiments}

The following numerical experiments are carried out under the Matlab software, using the mean square deviation (MES) to measure the signal denoising effect, the definition of mean square error generally defined as:

$$
M S E=\frac{1}{N} \sum_{i=1}^{N}\left(g_{i}-f_{i}\right)^{2}
$$

Which $f_{i}$ is the signal after denoising, $g_{i}$ is the original signal, it can be seen, after denoising the average variance is smaller, the better the denoising effect.

\subsection{The first numerical experiment}

This experiment uses Haar, db3, sym2, coif1, dmey wavelet base to denoise a sine signal, table 1 is the mean square error after denoising. Table 1 data can be obtained, the signal is suitable for a wavelet decomposition with dmey wavelet denoising, figure 1 and Figure 2 gives a section of the signal with dmey wavelet decomposition of a layer of noise figure.

Table 1 the mean square error of one-dimensional signal using soft wavelet threshold with different wavelet bases

\begin{tabular}{|c|c|c|c|c|c|}
\hline \multirow{2}{*}{ wavelet } & 1 & 2 & 3 & 4 & 5 \\
\cline { 1 - 5 } haar & 0.7132 & 0.7428 & 0.8340 & 0.9838 & 1.0090 \\
\hline db3 & 0.7070 & 0.7091 & 0.7722 & 0.9955 & 0.9984 \\
\hline sym2 & 0.7075 & 0.7150 & 0.7923 & 0.9872 & 0.9955 \\
\hline coif1 & 0.7073 & 0.7144 & 0.7910 & 0.9902 & 0.9967 \\
\hline dmey & 0.7069 & 0.7070 & 0.7083 & 0.9902 & 0.9919 \\
\hline
\end{tabular}




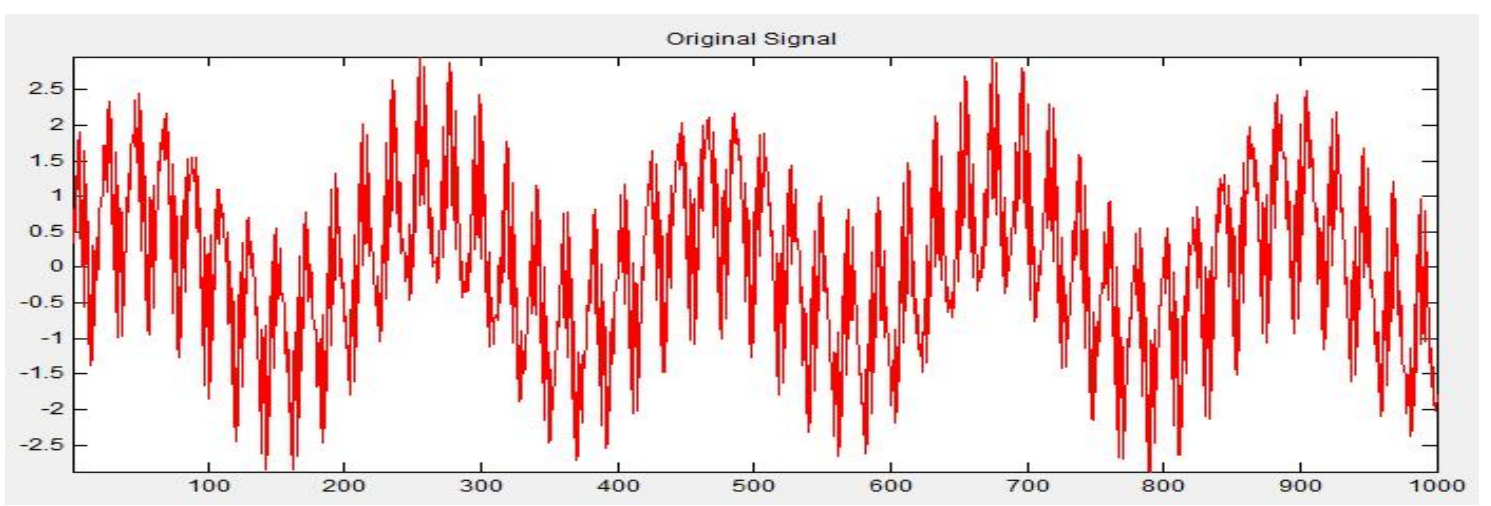

Fig. 1 Original signal

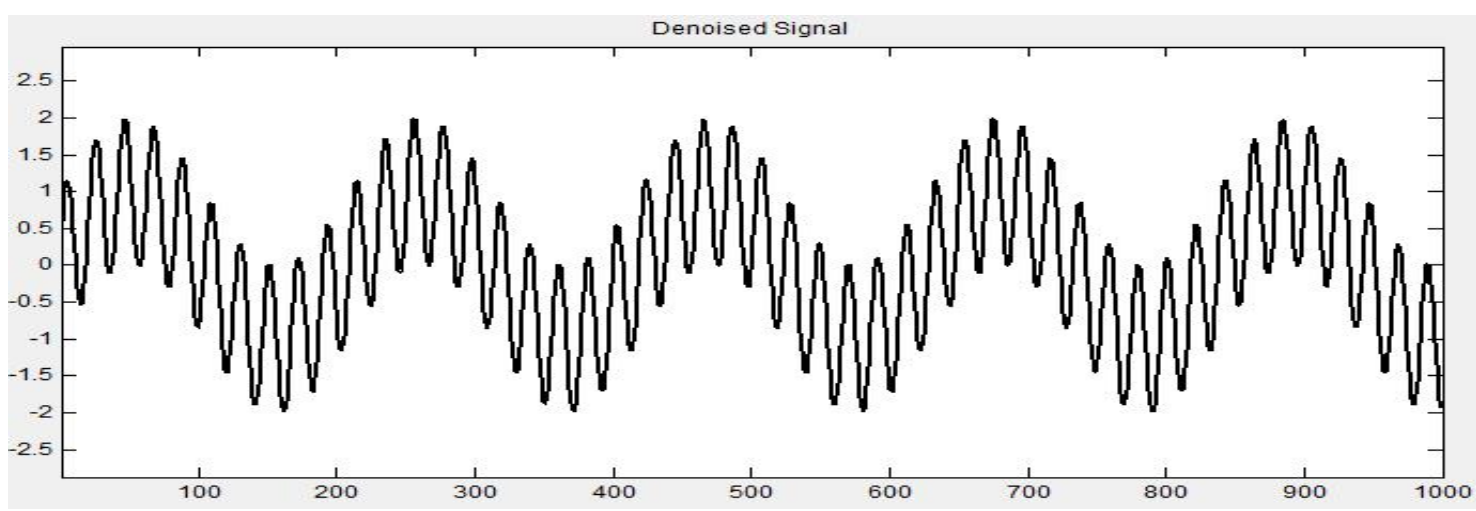

Fig. 2 Signal after denoising

\subsection{The second numerical experiment}

The one-dimensional signal containing Gauss white noise on a section of the use of $\mathrm{db} 3$ wavelet denoising, the decomposition layer is two,threshold function respectively take soft threshold and hard threshold function and threshold random, table 2 for the different situations on variance of noise, by the data in Table 2 can be obtained, for the one-dimensional signal denoising analysis, when the threshold is small, the better denoising effect; at the same threshold, soft threshold is better than the hard thresholding denoising.

Table 2 mean variance of different threshold function and threshold denoising

\begin{tabular}{|c|c|c|c|c|c|c|}
\hline Threshold & 0.7000 & 0.6000 & 0.5000 & 0.4000 & 0.3000 & 0.2000 \\
\hline $\begin{array}{c}\text { Soft } \\
\text { threshold }\end{array}$ & 0.1927 & 0.1913 & 0.1886 & 0.1741 & 0.1501 & 0.1135 \\
\hline $\begin{array}{c}\text { Hard } \\
\text { threshold }\end{array}$ & 0.1927 & 0.1816 & 0.1675 & 0.1358 & 0.0921 & 0.0561 \\
\hline
\end{tabular}

\section{Conclusions}

Based on wavelet analysis in one-dimensional signal denoising are studied, and the principle of threshold and threshold function of wavelet threshold denoising, the selection of wavelet base, decomposition layers and other related issues, and the relevant numerical experiments with Matlab software.

\section{References}

[1] Xin-lei Guo, Kai-lin Yang, Yong-xin Guo. Hydraulic Pressure Signal Denoising Using Threshold Self-learning Wavelet Algorithm[J]. Journal of Hydrodynamics, Ser.B, 2008, 20(4):433-439. 
[2] Chou Chien-Ming. A Threshoid Based Wavelet Denoising Method for Hydrological Data Modelling[J]. Water Resources Management, 2011, 25(7): 1809-1830.

[3] M. Nasri, H. Nezamabadi-pour. Image denoising in the wavelet domain using a new adaptive thresholding function[J]. Neurocomputing, 72(2009):1012-1025

[4] R. G. Yang, M. W. Ren. Wavelet denoising using principal component analysis[J]. Expert Systerms with Applications, 38(2011):1073-1076

[5] Michael T Johnson, Xiaolong Yuan, Yao Ren. Speech Signal Enhancement Through Adaptive Wavelet Thresholding[J]. Speech Communication, 2007, 49(2): 123-133.

[6] S. B, S. Papi. Filtered wavelet thresholding methods[J]. Journal of Computational and Applied Mathematics, 164-165(2004):39-52

[7] A. Azzalini, M. Farge, K. Schneider. A recursive method to determine the optimal denosing threshold[J]. Appl.Comput.Har Mon Anal, 18(2005):177-185

[8] Baussard. A, Nicolier. F, Truchetet. F. Rational multiresolution analysis and fast Wavelet Transform[J]. Application to wavelet shrinkage denosing Signal Processing, 84(2004):1735-1747

[9] M. Nasri, H. Nezamabadi-pour. Image denoising in the wavelet domain using a new adaptive thresholding function[J]. Neurocomputing, 72(2009):1012-1025

[10] S. Poornachandra. Wavelet-based denoising using subband dependent threshold for ECG signals [J]. Digital Signal Processing, 18(2008):49-55 